\title{
Humanidades digitales para el aprendizaje y difusión del Patrimonio Naval
}

\section{Digital Humanities for learning and dissemination of the Naval Heritage}

\author{
Lorena Martínez-Solís \\ Universidad de Murcia, Murcia, España \\ loremart@um.es \\ Celia Chaín-Navarro \\ Universidad de Murcia, Murcia, España \\ chain@um.es
}

\begin{abstract}
Resumen
Las prácticas innovadoras en la red en la enseñanza universitaria se consideran de gran interés, pero hay ámbitos académicos en los que es mucho más difícil ponerlas en marcha. Por ello nuestro objetivo fue llevarlas a la práctica docente, usando sistemas ubicuos, en un posgrado de Humanidades. Partimos de la base de que podría ser una forma nueva de aprender no sólo contenidos, sino también herramientas digitales, y de que estas competencias servirían para que los estudiantes adquirieran un importante grado de autonomía en la gestión de recursos virtuales, que además serían de gran utilidad en el mercado laboral. Para ello se eligieron las plataformas de mayor uso y adaptabilidad, como Wordpress para los blogs y Facebook y Twitter para las redes sociales. Los resultados de las encuestas confirman el elevado nivel de satisfacción por parte del alumnado y la percepción de que con este sistema han aprendido de forma diferente.
\end{abstract}

\section{Palabras Claves}

Innovación docente, e-innovación, aprendizaje ubicuo, Humanidades Digitales, Tecnología 2.0, docencia, posgrado, Máster, blog, redes sociales, web 2.0, Historia Naval, Patrimonio Naval.

\begin{abstract}
Innovating practices on the network at university education are considered with great interest, but there are academic areas where it is more difficult to implement them. Therefore, our goal was to bring them to teaching practice, using ubiquitous systems, in a Postgraduate Humanities. We assume that it could be a new way of learning not only contents, but also digital tools, and these competences would help students to acquire and important autonomy degree on the management of virtual resources, which would also be of great usefulness on the working market. To achieve it, we chose the platforms with greater use and adaptability, such as Wordpress for blogs and Facebook and Twitter for social networks. The results of the surveys confirm the high level of satisfaction on the part of the students and the perception that with this system they have learned in a different way.
\end{abstract}

\section{Key Word}

Innovation teaching, e-innovation, ubiquitous learning, technology 2.0, Digital Humanities, Teaching, Postgraduate, Master, blog, social networks, web 2.0, Naval History, Naval Heritage. 


\section{Introducción}

Las prácticas innovadoras en la enseñanza universitaria son uno de los temas que más interés despiertan, o al menos deberían hacerlo, entre los docentes. Desinterés, inercia, apatía y aburrimiento parecen diluirse en una clase cuando los estudiantes oyen algo que les suena a nuevos métodos de aprender. Al principio ni siquiera se lo creen, pero luego cuando ven que deben empezar una práctica diferente, la situación varía. Está comprobado que casi todos los nuevos sistemas, especialmente los vinculados con Internet suelen tener éxito, especialmente si hay detrás una planificación, seguimiento y rúbrica adecuados. Son múltiples los artículos que explican los buenos resultados, aunque casi todos concluyen que detrás del éxito hay mucho trabajo, tanto por parte de los estudiantes como de los docentes.

La puesta en marcha de alguna de estas innovaciones usando Internet tiene ritmos distintos dependiendo de los campos y áreas de conocimiento (Gross, 2016). Concretamente, en disciplinas experimentales (véase la revisión de Rojano, 2016) y en los grados de Educación, son múltiples las actividades que se llevan a cabo en este sentido, pero van descendiendo de manera acusada hasta llegar a las Humanidades. A pesar de ello, sí se han realizado algunas experiencias, como por ejemplo Mosaica (Barak, 2009), Bellas Artes (Abad, 2012), MUPATEC, proyecto de innovación sobre Museología (Fernández, 2015), y dentro de nuestro grupo de investigación (Martínez Solís, 2011 y 2015).

Hay muchos argumentos y explicaciones de este desfase en la tardía aplicación de las tecnologías a la docencia en estas áreas de conocimiento, aunque con una generación de nativos, o "cuasinativos", digitales la mayor parte quedan desautorizados, por lo que se pueden deber más a los docentes que a los discentes. Puede ser un tema de definición de competencias digitales del profesorado (Prendes 2018) o de que sea necesario que las propias instituciones docentes se impliquen más y apuesten de manera decisiva por ello.

Precisamente la falta de habilidades y de competencias en el alumnado en lo referido al mundo digital en los ámbitos de las Humanidades, y más concretamente de la Historia, hace que en el mercado laboral los titulados que además cuenten con estos conocimientos tengan muchas más opciones de conseguir un trabajo. Las Humanidades Digitales parecen haber resucitado el interés por incorporar la tecnología a los procesos de aprendizaje e investigación, pero precisamente la Historia es una de las ramas menos desarrolladas de este ámbito académico.

Ya disponemos de modelos sobre las habilidades digitales al alcanzar, como DigComp 2.1., que es el marco de referencia sobre cuáles deben ser las competencias de los ciudadanos en este nuevo ambiente virtual, y marca 8 niveles, desde uno inicial hasta el de mayor grado de especialización (Carretero, 2017). Valverde-Crespo (2018) divide estas

Humanidades digitales para el aprendizaje y difusión del Patrimonio Naval. Lorena Martínez-Solís y Celia Chaín-Navarro

Página 2 de 19 
competencias en cinco áreas: información, comunicación y colaboración, creación de contenido digital, seguridad y resolución de problemas.

Estos argumentos fueron una de las bases para poner en marcha una práctica innovadora que despejara algunas incógnitas sobre la adecuación de introducir Internet en la docencia de las Humanidades. El avance de la red y la propia evolución de las prácticas e-innovadoras nos han permitido implementar una serie de instrumentos que hace 10 años hubiera sido impensable, así podemos incorporar a la docencia presencial una serie de complementos. Sin embargo, existía un importante condicionante: la bibliografía previa avisaba de que la planificación previa y la monitorización in situ requerían invertir mucho tiempo, aunque garantizaban un mayor porcentaje de éxito, afirmaciones que luego pudimos comprobar que eran ciertas.

Por todo ello decidimos hacerlo de manera planificada (con un diseño instruccional previo), integrada (se usan distintos programas, pero se aprende a utilizarlos de manera coordinada y unificada), con algunas de las herramientas más usadas a nivel mundial (blog y redes sociales) que están perfectamente preparadas para utilizarse tanto en ordenadores como con dispositivos móviles, y llevando a cabo una evaluación formativa (sendas rúbricas) y sumativa (seguimiento y evaluación continua de cada estudiante) que consideramos adecuada.

La otra base sobre la que nos asentamos fue nuestra experiencia con estas herramientas, ya que desde hace años gestionamos el blog (https://blogcatedranaval.com/) y los perfiles en redes sociales (https://www.facebook.com/catedranaval/ y https://twitter.com/catedranaval), de la Cátedra de Historia y Patrimonio Naval (en adelante HyPN), institución que coordina el Máster en el que pusimos en marcha este proyecto, así como actividades sobre e-innovación realizadas anteriormente en otras materias, aunque mucho menos complejas (Chaín, 2008).

El área concreta de aplicación es la Historia Naval y Marítima, un ámbito muy poco desarrollado académicamente, y que aunque a primera vista parezca minoritario, realmente hay muchas personas que por diferentes motivos (familiares, académicos, legales, patrimoniales) están muy interesadas en él. La prueba del interés que despierta es precisamente el número de visitas que las herramientas digitales de la Cátedra de Historia y Patrimonio Naval tiene a diario, que pueden oscilar entre las 1000 y los 2000, desde más de 50 países distintos y de múltiples idiomas nativos.

Los motivos arriba indicados nos llevaron a poner en marcha una experiencia nueva en el ámbito disciplinar de la Historia, en un posgrado con titulados de diferentes lugares de España y algunos de otros países americanos y europeos, precisamente para poder analizar con más alcance el éxito de esta e-innovación. El objetivo de la práctica docente era doble: enseñar unos conocimientos de gestión de contenidos digitales, que podían abrir a los

\footnotetext{
Humanidades digitales para el aprendizaje y difusión del Patrimonio Naval. Lorena Martínez-Solís y Celia Chaín-Navarro

Página 3 de 19
} 
estudiantes las puertas en el mercado laboral y también evaluar el éxito de la propuesta, en especial conocer si los estudiantes piensan que así se aprende de manera diferente. Por otro lado, no podíamos obviar la necesidad existente en Internet, constatada por nuestra experiencia, de publicar contenidos sobre Historia y Patrimonio Naval, lo que también a medio plazo se puede convertir en una salida profesional.

De manera muy resumida, esta e-innovación se basa en un principio básico: nuestro objetivo es que los estudiantes aprendan, y si existen herramientas que facilitan el aprendizaje son las que usaremos. Pero las aplicaciones en sí nunca son el objetivo, sino los medios para conseguir lo propuesto, siempre que, evidentemente, éstas mejoren la situación de partida. Como se ha dicho en el caso de las Humanidades Digitales, hasta que no dejemos de escribir sobre ellas, las tecnologías no estarán integradas. Mientras, en lugar de ser concebidas como lo que son, un medio para alcanzar un fin, podrán considerarse tanto un problema como una solución (Chaín, 2013; Onrubia, 2016).

En este sentido las tecnologías ubicuas aplicadas a la enseñanza tienen el mismo fin: acercarse al alumnado en cualquier sitio y a través de cualquier medio con el que pueda conectarse, para que facilitando el acceso el proceso de aprendizaje, éste se lleve a cabo de una manera más natural y sencilla, casi sin que sea consciente de ello porque se siente motivado e interesado.

\section{La aplicación de tecnologías en la enseñanza universitaria: los blogs y las redes sociales. Breve estado de la cuestión}

Muchos han sido los autores que han escrito sobre las ventajas e inconvenientes de la aplicación de las TICs en la educación universitaria, de las posibilidades de la Web, de la siguiente generación de herramientas 2.0 como las redes sociales o el resto de los entornos colaborativos (blogs, wikis...).

La literatura científica ha otorgado tanto a los blogs como a las redes sociales que se utilizan en la enseñanza una serie de ventajas, tales como capacidad para aumentar la interactividad y la colaboración (Colas Bravo, 2015; Halic, 2010; Morón, 2016), promover la autoexpresión (Halic, 2010), exige el desarrollo de múltiples habilidades y estrategias (Gotkas, 2009), permite un participación activa y desarrolla el pensamiento crítico (Morón, 2016), mejoran la percepción del aprendizaje y la motivación (Chaín, 2008; Churchill, 2009 y Halic, 2010), se adaptan a cualquier materia y ciclo académico (Morón, 2016), permiten crear una identidad digital e impulsan la creatividad (Colas, 2015), actitud positiva del alumnado frente a ellas, facilidad de uso, vastas posibilidades comunicativas de estos

\footnotetext{
Humanidades digitales para el aprendizaje y difusión del Patrimonio Naval. Lorena Martínez-Solís y Celia Chaín-Navarro

Página 4 de 19
} 
canales (especialmente las redes sociales) (Gómez y Roses, 2012) y que permiten una evaluación continua (Morón, 2016), entre otros.

Centrándonos en el uso de bitácoras, podemos considerar que nos encontramos en una segunda fase con respecto a la introducción de los blogs en la enseñanza universitaria. Tuvimos una etapa inicial de teorizar sobre el tema, que no está del todo acabada por la propia naturaleza de la herramienta, y una segunda de exploración mediante la experimentación, o lo que es lo mismo, planificar la actividad, llevar a cabo la práctica, evaluarla y comunicar los resultados a la comunidad científica. También se publican revisiones de experiencias ajenas (Salgado, 2013). En esta segunda etapa, los trabajos experimentales realizados hasta el momento con blogs se pueden incluir en estas categorías:

- Blogs desarrollados por los docentes en los que el alumnado debe/puede intervenir (por ejemplo: Fernández, 2015; Krause, 2005; Maquilón, 2017 y Molina, 2016a). Son los que primero aparecieron en el universo docente.

- Blogs que deben realizar los alumnos como tarea de la materia/asignatura, con rúbrica docente o sin ella, con temas del curso (Hernández, 2004). Las bitácoras se pueden implementar por parte de los discentes de manera autónoma o grupal (Goktas, 2009; Halic, 2010; Martín, 2017; Top, 2012 y Rojano, 2016). Suelen ser los más efectivos, aunque suponen invertir mucho tiempo en la planificación, desarrollo, seguimiento y evaluación.

- Mixto: Dapía, 2014 (blog maestro con blogs satélites) y Molina, 2013 (varias experiencias unidas, unas con blogs de docentes y otras de alumnos).

Son muy pocas las prácticas con Wordpress (Maquilón, 2017) y mínimo el número de los que integran bitácora y redes sociales (una excepción es Morán, 2016). En el ámbito de las Humanidades posiblemente sea la primera.

Finalmente, cabe destacar dos tesis. Por un lado, la de Abad (2012), en la que realiza un profundo recorrido sobre muchas de estas herramientas, las prueba y lleva a cabo una selección de las considera mejores, y, por otro, la de Morán, defendida en 2016. Con esta última, el autor pretende consolidar: "un modelo de software que, en combinación con un portal docente y un sistema de blogging de las asignaturas usando Wordpress y de red social basada en Facebook y Twitter, posibilite la interacción constante del alumnado desde cualquier plataforma con un programa que facilita la conexión directa a esas y otras herramientas". Tema estrechamente relacionado con el que nosotros presentamos en este trabajo, ya que se utilizan las mismas aplicaciones y se persiguen prácticamente los mismos fines iniciales.

En cuanto al uso de las redes sociales en la metodología docente universitaria, han visto la luz, en los últimos cinco años, varias y diversas publicaciones realmente interesantes. El crecimiento de investigaciones en este sentido ha sido exponencial,

Humanidades digitales para el aprendizaje y difusión del Patrimonio Naval. Lorena Martínez-Solís y Celia Chaín-Navarro

Página 5 de 19 
produciéndose en el último año la mayoría de las mismas y, en casi todas se habla del uso de Facebook como herramienta docente. Algunos de estos trabajos son los de Wong (2017), Romero (2017), Payá (2016 a y b), González Martínez (2016), Cabrera (2016), Albertos (2016), Martínez Serrano (2016), González Bencomo (2016), Arguedas (2016), Sánchez Rodríguez (2016), Jiménez Migallón (2016), Rovira (2016) y González Ramírez (2016), entre otros.

Las humanidades digitales, el amplio campo de acción en el que se enmarca esta práctica, es una de las áreas sobre las que se habla mucho, se escribe, pero apenas se pone en práctica. En los últimos veinte años, como bien mantiene Spence (Romero-Frías, 2014), "ha pasado de ser un espacio para la experimentación tecnológica en aplicaciones literarias y lingüísticas, a convertirse en un campo que abarca casi todas las áreas tradicionales y emergentes de las humanidades, que se aprovecha de cualquier innovación tecnológica aplicable y que funde procesos de creación y construcción/representación con una reflexión amplia y profunda sobre los efectos de la tecnología en la enseñanza y la investigación científica”. Una revisión de este panorama se puede encontrar en Iribarren (2017).

De nuevo volvemos a la necesidad de la integración de las nuevas posibilidades que brinda Internet a un ámbito tradicionalmente alejado de las tecnologías, pero que en el nivel discente va dirigida a una generación más habituada a moverse entre recursos virtuales. Ello brinda la posibilidad de crear espacios de afinidad entre estudiantes y profesores, además de potenciar y ampliar una de las funciones básicas de las Humanidades: interpretar el impacto sociocultural de esas tecnologías, entendidas éstas como bienes, como creaciones, aplicaciones y respuestas de esas tecnologías a las preguntas culturales, sociales, históricas y filológicas planteadas (Chaín, 2013).

Centrándonos en el tema del uso de las tecnologías de la información y comunicación en los estudios de tercer ciclo, hemos detectado que en los últimos cinco años se han publicado, sólo en España, más de una veintena de trabajos interesantes al respecto. En todos ellos, sus autores cuentan sus experiencias u opiniones sobre esta cuestión. Desde nuestro punto de vista, las obras más relevantes son las de Marcelo, Rivero, Martínez Clares y Linares.

Dentro de "la teoría del aprendizaje con las tecnologías" destacamos, porque su punto de vista sirve de marco de referencia para nuestra experiencia, las palabras de Reigeluth (2012) sobre el nuevo paradigma de instrucción. Según el autor, entre los nuevos roles para los docentes se incluyen los de diseñador del trabajo del alumno, facilitador del proceso de aprendizaje y mentor atento. Por su parte, para los estudiantes se incluyen: trabajador, estudiante autónomo y co-instructor.

Humanidades digitales para el aprendizaje y difusión del Patrimonio Naval. Lorena Martínez-Solís y Celia Chaín-Navarro

Página 6 de 19 


\section{El escenario}

El Máster oficial en Historia y Patrimonio Naval es un posgrado anual que ofrece una formación multidisciplinar especializada en los ámbitos históricos Naval y Marítimo. Permite que los egresados puedan escoger entre varias opciones profesionales en diferentes instituciones, en las que podrán gestionar el Patrimonio vinculado a nuestro pasado en el mar. Las disciplinas que se imparten estos estudios de tercer ciclo son Historia, Documentación, Arqueología Subacuática, Comunicación, Derecho y Disciplinas Auxiliares. Este título se ha caracterizado desde su puesta en marcha, entre otras cuestiones, por implementar y evaluar la utilización de las tecnologías y los entornos virtuales de enseñanza como apoyo en el aprendizaje y la innovación en el mismo.

Una de las materias en las que estas cuestiones se han intensificado ha sido en la denominada Técnicas documentales para la conservación y gestión del Patrimonio Histórico Naval, asignatura troncal anual de 12 créditos. A grandes rasgos, los contenidos impartidos en ella son: Conservación y Restauración, Archivística técnica avanzada y archivos electrónicos, Biblioteconomía y Recuperación de información para la conservación y la gestión patrimonial. En ella, una parte de la formación consiste en que los alumnos generen y mantengan durante todo el curso un blog y dos perfiles en redes sociales, concretamente en Facebook y en Twitter.

Aunque los objetivos docentes eran menos ambiciosos, tanto por los resultados de esta actividad con el alumnado como por la propia experiencia profesional de las docentes, y basándonos en bibliografía previa existente al respecto, nos hemos propuesto averiguar si realmente la tarea de aprender a difundir lleva consigo una "nueva forma de aprender". Y con esta frase nos referimos más a la percepción que el alumnado tiene de que aprende de manera distinta, que porque realmente marque una diferencia sustancial en la forma de adquirir conocimiento. Sin embargo, esta percepción mejora el interés y la motivación, permite a los docentes interactuar desde una perspectiva ventajosa y contribuye a generar un depósito de conocimiento adquirido de mucho interés. Es también una forma de convertir a los estudiantes de estas áreas de Humanidades en prosumidores, lo que les otorga autonomía y capacidad para moverse en el universo virtual con suficientes conocimientos para analizar y decidir qué es lo que les interesa, con qué se quedan y cómo lo difunden.

En referencia a la planificación y rúbrica (que tienen disponibles desde el inicio del curso en el aula virtual), en primer lugar se imparten varias clases magistrales explicando las herramientas y su uso. En la primera se les explica a los alumnos qué es el Patrimonio, por qué es importante difundirlo (sólo se puede proteger aquello que se conoce) y a través de qué vías puede hacerse (entre ellas las que van a conocer en la práctica). En la segunda \begin{tabular}{l}
\hline $\begin{array}{l}\text { Humanidades digitales para el aprendizaje y difusión del Patrimonio Naval. Lorena Martínez-Solís } \\
\text { y Celia Chaín-Navarro }\end{array}$ \\
Página 7 de 19
\end{tabular} 
aprenden qué es el community management y cuáles son las funciones del community manager. Asimismo, y desde la experiencia profesional en el sector, se les ofrecen una serie de pautas y consejos: constancia, regularidad y calidad de las publicaciones; predisposición, empatía y paciencia con el usuario; técnicas para aumentar el número de seguidores; herramientas de gestión útiles y dónde encontrar las fuentes más importantes.

Se le da al alumnado dos semanas para que busquen un tema dentro de las áreas del Máster que sea de su interés para el desarrollo de las herramientas 2.0. Ese mismo día la profesora les ayuda a crear los perfiles, aunque por lo general no suelen tener problemas para hacerlo $\mathrm{y}$, a partir de ese momento, se hace un seguimiento casi diario de las publicaciones por parte de los alumnos. A la semana siguiente se les explica lo que son las bitácoras y los gestores de contenido, y qué es y cómo funciona uno de ellos: Wordpress. Sobre el blog hay tres sesiones teóricas en las que se va explicando poco a poco (a la vez que ellos van construyendo sus bitácoras) funcionalidades y herramientas.

A lo largo del curso se resuelve cualquier tipo de duda y se ofrecen tips relacionados con la gestión. Las entradas que se publican en el blog se difunden también en las redes sociales, con lo que el número de visitas a la bitácora se multiplica. Para los perfiles de Facebook y Twitter es una buena baza disponer de entradas sobre el tema elegido que se publican cada 10 o 15 días, con lo que pueden integrar los esfuerzos dedicados en ambos sistemas.

Al final se evalúan las herramientas, tarea relativamente sencilla dado que a lo largo del año académico se observa la trayectoria de las mismas: para los blogs las entradas, su calidad, fuentes utilizadas, inclusión de herramientas multimedia y uso de la barra lateral, entre otras. Para las redes sociales cuándo y cuántas publicaciones se hacen, tipología (texto, imágenes o vídeo), cantidad de "me gusta" y "retweets" que reciben, fans y followers que tienen los perfiles, etc.

En referencia a cómo se evalúa la asignatura, los puntos se reparten del siguiente modo: dos, como máximo, por cada uno de los grandes bloques (Archivística, Biblioteconomía, Museología y Documentación), y los otros dos por el desarrollo de las herramientas 2.0 (hasta 1 punto por la gestión de la bitácora y hasta 1 punto por la de los perfiles en redes sociales (Facebook y Twitter). El mejor de cada uno de ellos obtiene un punto adicional.

\section{Materiales y Métodos}

Las herramientas utilizadas fueron las de mayor uso que, además, presentaban una óptima adaptación para dispositivos móviles, tanto para la creación y desarrollo de la bitácora como de las redes sociales. \begin{tabular}{l}
\hline $\begin{array}{l}\text { Humanidades digitales para el aprendizaje y difusión del Patrimonio Naval. Lorena Martínez-Solís } \\
\text { y Celia Chaín-Navarro }\end{array}$ \\
Página 8 de 19
\end{tabular} 
Se usó Wordpress porque es el sistema de gestión de contenidos más utilizado en el mundo profesional, gratuito, fácil de usar para estudiantes con pocos conocimientos tecnológicos que además proporciona muchas opciones, tiene enormes capacidades para la estructuración y jerarquización de la información (habilidades y competencias que se incluyen en la asignatura) y cuenta con una de las mejores adaptaciones a los dispositivos móviles desde hace años. Existen plataformas similares como Joomla o Drupal, pero una vez estudiadas se vio que podían presentar serios problemas para estudiantes con unas habilidades tecnológicas reducidas.

La bibliografía deja claro que muchas de las prácticas innovadoras con bitácoras se han llevado a cabo con otras plataformas, que aparentemente son más sencillas de aprender como Blogger o Blogspot, pero la funcionalidad de Wordpress para indizar contenidos, crear categorías y etiquetas y su adaptabilidad a los nuevos dispositivos hacía que fuera el más idóneo.

En el apartado de las redes sociales elegimos Facebook y Twitter porque, año tras año, según diversos estudios, están entre las aplicaciones 2.0 más populares entre los usuarios. Además, dadas sus características intrínsecas, el conocimiento y manejo de ambas herramientas favorecen que la formación de los estudiantes sea más completa.

Contamos con un diseño instruccional previo que es el resultado de una planificación iniciada un año antes, y que se basa por una parte en nuestra experiencia en el tema, tanto docente como profesional de uso de estas herramientas y, por otra, en la bibliografía consultada y en las reuniones mantenidas para lograr llevar a cabo con éxito la práctica.

Una de las características de esta metodología ha sido la integración de dos de las plataformas más utilizadas en la web 2.0, que se coordinan para lograr una mayor difusión y por tanto el éxito de la práctica. Ambas están perfectamente preparadas para utilizarse tanto en ordenadores como con dispositivos móviles, con lo que el estudiante puede hacer uso de ellas y gestionarlas desde cualquier dispositivo con conexión a Internet. Se han llevado a cabo dos tipos de evaluación; una formativa (sendas rúbricas de cada una de las herramientas, disponibles desde el primer día para el alumnado, así como varias horas de clase sobre en el uso de ambas herramientas) y otra sumativa (para ello se utilizaron además otras herramientas que estaban disponibles a través el Aula Virtual de la Universidad de Murcia, como la wiki, que es como una pizarra electrónica y el mini-blog, que se usó para incluir lecciones sobre temas que las docentes constataron que no se habían quedado muy claros).

Para cumplir con el objetivo de conocer si los alumnos del Máster en Historia y Patrimonio Naval consideran que han aprendido nuevas técnicas para gestionar este tipo de herramientas 2.0, si les está siendo (o les va a ser) útil a nivel profesional, si recomiendan

\footnotetext{
Humanidades digitales para el aprendizaje y difusión del Patrimonio Naval. Lorena Martínez-Solís y Celia Chaín-Navarro

Página 9 de 19
} 
su uso como material de apoyo en otras asignaturas de este o de otros posgrados, si creen que son beneficiosas para dar a conocer la Historia y/o el Patrimonio y la Historia Naval y Marítimo y, más específicamente, si consideran esta forma de aprendizaje algo diferente, les enviamos un cuestionario, a través de una aplicación de Internet denominada "Encuestas". Esta herramienta ha sido desarrollada por la Universidad de Murcia, garantiza el anonimato, permite rellenar el formulario desde cualquier dispositivo conectado a la red y facilita la obtención y análisis de los resultados. Se enviaron 37 encuestas (una por egresado) y fueron cumplimentadas el $62 \%$.

\section{Resultados}

Desde hace años, el profesorado de la Cátedra apuesta tanto por el uso de las herramientas 2.0 en la docencia universitaria (Chaín, 2008), como para su utilización en la difusión de la Historia y el Patrimonio Naval (Chaín, 2013; Martínez Solís, 2011 y 2015). Dados los buenos resultados que siempre hemos obtenido, creímos necesario impartir este tipo de conocimientos en los ámbitos de las Humanidades, y el Máster en Historia y Patrimonio Naval que se puso en marcha en el curso 2013/2014 era un buen escenario de prueba. Nuestro objetivo al incluir una e-innovación era múltiple: comprobar si unos titulados en Humanidades eran capaces de aprender a gestionar contenidos en la web 2.0 de la misma manera que ya lo habían hecho alumnos titulados de otras áreas de conocimiento, dotar a los estudiantes de unos conocimientos actualmente muy demandados en cualquier institución pública o privada que les fueran de utilidad a nivel profesional; impartir una enseñanza innovadora que fuera capaz de motivar a los discentes; así como dar a conocer y contribuir a poner en valor nuestro rico y extenso Patrimonio Naval y Marítimo. Las respuestas han sido afirmativas en todos los casos, y este resultado es tanto por la parte docente como por la discente.

Para poder constatar si se habían alcanzado los objetivos propuestos, aparte del análisis por parte de las docentes y de contrastar opiniones con otros profesores, también se entrevistó al alumnado de las tres promociones finalizadas, porque eran una pieza clave en la valoración de esta e-innovación, ya que desde el primer momento se les exigía una cierto nivel de autonomía que con el tiempo se transformó en independencia a la hora usar las plataformas, de buscar fuentes, analizarlas, sintetizarlas, redactar noticias y/o entradas y publicarlas. Este esquema de trabajo en el que el estudiante puede controlar su aprendizaje responde a los requisitos sobre el cambio educativo descritos en Caro (2018).

Las características sociológicas de los estudiantes que han participado en el estudio son muy similares al perfil del alumnado global del posgrado (hombres, menores de 30 años y asistencia habitual a las clases). Por lo general contaban con conocimientos básicos o nulos en materia de gestión de blogs y redes sociales antes de empezar el Máster. La

\footnotetext{
Humanidades digitales para el aprendizaje y difusión del Patrimonio Naval. Lorena Martínez-Solís y Celia Chaín-Navarro

Página 10 de 19
} 
media de las visitas recibidas en los blogs que elaboraron fue de 6.500 y el número de fans fue de 675 en Facebook y de 520 en Twitter, todo ello en sólo 6 meses.

Del análisis general destacamos que lo que más les ha gustado a los alumnos de trabajar con las herramientas 2.0, ha sido profundizar/investigar en temas de su interés y, sobre todo, interactuar con los lectores/seguidores. Lo menos gratificante para ellos, sin embargo, ha sido la inversión de tiempo que han tenido que hacer: en el blog a la hora de redactar las entradas que, aunque sólo eran 15, debían tener "consistencia"; en las redes sociales porque tenían que publicar a diario para mantener vivos los perfiles. A día de hoy, el 35\% continua gestionando su bitácora (porque "constituye una fuente muy interesante para desarrollar mis conocimientos, no sólo de Historia Naval, sino en cuanto a los referentes a la difusión entre el público, algo que considero básico"; "es un medio excelente de poder compartir un conocimiento adquirido y aplicar métodos de investigación enseñados"; por "interés personal y profesional"; "me satisface personalmente"; "es entretenido y reconforta ver que la gente me sigue") y los que no lo hacen argumentan exclusivamente falta de tiempo. Sin embargo, el 70\% aún mantiene vivos los perfiles de las redes sociales (les parece que "es sencillo de manejar, gratificante, rápido, divertido y ameno"; también por interés personal y profesional; "para seguir informando a mis seguidores sobre mi tema, a diario si es posible"; "me gusta compartir en las redes sociales material histórico").

No deja de ser curioso que la opinión de los estudiantes sobre el tiempo que supone tener que gestionar cada una de las herramientas les llevaba bastante $(52,2 \%$ para el blog y $47,8 \%$ para redes sociales) o mucho tiempo ( $26,1 \%$ y $21,7 \%$ respectivamente), cuando realmente una vez que se conoce el manejo de las redes sociales y se tienen localizadas las fuentes de información, se requiere mucho menos tiempo que para redactar una entrada en el blog. Posiblemente el hecho de que tuvieran que publicar a diario les causaba mayor zozobra, pero una vez pasado el tiempo los porcentajes de los que todavía mantienen las herramientas aprendidas (las redes sociales duplican los blogs vivos) confirman que la bitácora requiere mucha más dedicación.

En general, la mayoría opina que los conocimientos aprendidos en materia 2.0 han sido útiles para su desarrollo profesional (4 sobre 5), están muy satisfechos con la experiencia (4,1 sobre 5), todos recomiendan el uso de estas herramientas como material de apoyo en otras asignaturas de este o de otros posgrados y el $95 \%$ piensa que son útiles para dar a conocer la Historia y/o el Patrimonio y la Historia Naval y Marítima.

Del análisis más concreto que hace referencia al autoaprendizaje, el 95,7\% ha contestado que sí, que habían aprendido de forma distinta. Otra cifra que sirve de base a esta afirmación es que con una media de 4,3 sobre 5, la mayor parte de los encuestados admitió que había aprendido más de HyPN con este sistema.

\footnotetext{
Humanidades digitales para el aprendizaje y difusión del Patrimonio Naval. Lorena Martínez-Solís y Celia Chaín-Navarro

Página 11 de 19
} 


\section{Conclusiones}

Los resultados obtenidos evidencian la necesidad del cambio de perspectiva, la evolución del rol de aprendizaje pasivo a otro más motivante, que es que los discentes tengan en sus manos la posibilidad de aprender para hacer algo útil: transmitir lo aprendido. Aprender para dar a conocer, obtener conocimiento lo antes posible para que otros también lo conozcan.

El hecho de tener que difundir supone que el tiempo de aprendizaje se debe limitar, lo que como factor positivo implica lograr mucho antes un grado importante de autonomía o independencia en esa temática. Además, los estudiantes, al estar pendientes de escribir para otros sienten que aprenden de una manera distinta. También, aparte, obtienen un feedback relativamente rápido a través de las redes sociales, lo que les motiva a seguir.

Estas prácticas hacen que el proceso de aprendizaje sea más corto, porque está espoleado por el de difundir (y en este sentido entiéndase enseñar a otros) y nos dirige hacia la consideración de un empoderamiento discente en los procesos de adquisición de conocimiento, a alcanzar esa independencia tan destacada en las competencias digitales, que es muy importante en un posgrado.

Otros trabajos previos en los que se preguntaba a los estudiantes su opinión acerca de este tipo de prácticas sobre competencias digitales (ya sean blogs o redes sociales, o incluso el portafolios) ofrecen unos resultados similares, con cifras que están por encima del $80 \%$ en el nivel de satisfacción y más del $90 \%$ recomiendan que se apliquen en otras asignaturas. En estos análisis se destaca de nuevo la interactividad y facilidad de las herramientas, con las que están mucho más familiarizados que la generación anterior, así como la oportunidad de opinar, crear contenidos y de diseñar recursos (Abad, 2012; Chaín, 2008; Molina 2016b; Moreno Fernández, 2017 y Martín-Montilla, 2016).

Así, de los niveles marcados DigComp 2.1 (Carretero, 2017), el marco de referencia europeo sobre las competencias digitales de los ciudadanos, esta experiencia alcanza los dos últimos, los más altos, ofreciendo a los titulados una elevada especialización (el nivel 7 se corresponde con la habilidad de resolver tareas complejas con soluciones previstas y el 8 resolver problemas complejos en los que interactúan diversos factores), ya que aprenden a localizar, seleccionar y analizar recursos y posteriormente a crear los suyos propios, utilizando una herramienta de fácil manejo pero de amplísimo alcance y repercusión Se cumplen también las cinco áreas previstas por Valverde-Crespo (2018) en este tema.

Y aunque apenas se ha escrito sobre ello, estas prácticas en entornos virtuales les sirven a los discentes para retomar algo de la capacidad creativa que tienen y que el sistema educativo actual, en nuestra opinión, adormece.

Humanidades digitales para el aprendizaje y difusión del Patrimonio Naval. Lorena Martínez-Solís y Celia Chaín-Navarro

Página 12 de 19 
En el esquema del modelo de competencia docente holística para el mundo digital en la enseñanza básica que propone Esteve (2018), nosotras añadiríamos otro, más propio de los niveles universitarios, sobre la sensibilidad del docente, que también debe transmitir al discente, acerca de la necesidad de valorar y de difundir el patrimonio cultural, o cualquier otra materia sobre la que se aplique esta e-innovación.

Otra ventaja de enseñar competencias digitales a los estudiantes de humanidades es que no sólo les brindamos más posibilidades dentro de esta sociedad de la información, sino que estamos generando expertos con suficientes conocimientos para que se conviertan en masa crítica sobre estas tecnologías que usan y conocen muy de cerca, una de las metafunciones de las Humanidades Digitales.

Para el docente hay veces que suele ser un problema enfrentarse a algún estudiante que no quiere hacer el blog o las redes sociales, pero por experiencia sabemos que, a posteriori, son de los que más entusiasmados están con las herramientas que han generado.

Si hubiera que resaltar un aspecto problemático es, sin duda, que hay que invertir mucho tiempo, tanto por parte del docente para planificar la práctica, generar una rúbrica válida y monitorizar de manera personalizada el desarrollo de las herramientas, como por la de los estudiantes, como ha puesto de manifiesto los resultados de la encuesta, que deben invertir en comenzar a usar la herramienta, buscar contenidos, asimilarlos, resumirlos, redactar las entradas y darles difusión en las redes sociales. Sin embargo, adquirir conocimiento, difundirlo y conseguir que los usuarios de esa información respondan, no son una actividades ni mucho menos rápidas, y sin embargo con las herramientas $2.0 \mathrm{se}$ convierte en un ejercicio integrado que proporciona nuevos saberes tanto a los estudiantes como a sus lectores, sin dejar atrás lo que en este proceso aprendemos los docentes (de la forma intuitiva de aprender el software de los alumnos, de cómo actúan preprofesionalmente en la red, de su ingenio y creatividad, y sobre todo de cómo está cambiando la nueva generación de estudiantes en sus procesos de aprendizaje).

Por todas estas razones, con las ventajas e inconvenientes de la herramienta, creemos que favorece no sólo la adquisición de competencias digitales, sino también el desarrollo integral del alumnado al unir en una sola faceta búsqueda de datos y de información, análisis y síntesis de los contenidos para hacer una entrada, corrección del texto, indización/categorización de la entrada. Es decir, que acaba en que el estudiante consigue tener un conocimiento de un tema del que antes apenas sabía nada, y este conocimiento recién adquirido es mucho más duradero que el que se consigue estudiando para un examen. Es, además, conocimiento adquirido con la práctica y su resultado tiene una utilidad social clara (difusión del patrimonio). El uso de los contenidos en abierto que ofrece Internet, además de enriquecer el sistema de enseñanza-aprendizaje, modifica la propia investigación que pasa a ser producida de manera global y colaborativa (Gros, 2012).

Humanidades digitales para el aprendizaje y difusión del Patrimonio Naval. Lorena Martínez-Solís y Celia Chaín-Navarro

Página 13 de 19 
Esta experiencia, en la que no es necesario invertir recursos económicos, es aplicable en cualquier lugar, ya que sólo hay que tener acceso a la red, conocer las herramientas (blog y redes sociales) y estar dispuesto a dedicar tiempo y esfuerzo a la actividad, tanto previamente, para la planificación, como durante su puesta en marcha. Además, conseguirá tener un doble feedback (estudiantes e internautas) y generar una base de conocimiento pública muy importante para el análisis, la evaluación de la experiencia y para el rediseño, en caso de que éste sea necesario. Los resultados no sólo son gratificantes para docentes y discentes, sino que, al ser públicos, contribuyen a dar a conocer un patrimonio global, que une e identifica a todos los pueblos que se han desarrollado cerca del mar. Además, dando un salto hacia otros sectores, los productos resultantes, los blogs de los estudiantes, sirven para aumentar la cobertura de la economía azul y de uno de los segmentos económicos más en auge en la actualidad: el turismo. Esto contribuye directamente a proporcionarles herramientas, les ayuda a generar su identidad virtual y ofrece salidas profesionales alternativas para que puedan acceder al mercado laboral en un tiempo más corto.

Presentación del artículo: 28 de abril de 2017

Fecha de aprobación: 23 de marzo de 2018

Fecha de publicación: 31 de marzo de 2018

Martínez-Solís, L. y Chaín-Navarro, C. (2018). Humanidades digitales para el aprendizaje y difusión del Patrimonio Naval. RED. Revista de Educación a Distancia, 57. Recuperado de: http://www.um.es/ead/red/57/martinez_chain.pdf

\section{Financiación}

El presente trabajo se inserta en el proyecto de investigación denominado "Nauticum" (19496/PI/14), financiado por la Fundación Séneca - Agencia de Ciencia y Tecnología de la Región de Murcia.

\section{Referencias bibliográficas}

Abad Gómez, R. (2012). La web 2.0 como herramienta didáctica de apoyo en el proceso de enseñanza aprendizaje: aplicación del blog en los estudios de Bellas Artes (tesis doctoral). Universidad Complutense de Madrid.

Albertos San José, A., Domingo Roget, À. y Albertos San José, J.E. (2016). Estrategia docente para el desarrollo de la competencia digital en el aula universitaria. Del uso recreativo al uso formativo. Educar, 52(2), pp. 243-261. Recuperado de

\footnotetext{
Humanidades digitales para el aprendizaje y difusión del Patrimonio Naval. Lorena Martínez-Solís y Celia Chaín-Navarro

Página 14 de 19
} 
https://goo.gl/gUhwwD

Arguedas-Méndez, S.M. (2016). El Facebook como apoyo a la docencia universitaria. Experiencia educativa en un Curso de Cálculo. Revista electrónica Educare, 20(1). Recuperado de https://goo.gl/WJADAG

Barak, M. [et al.]. (2009) MOSAICA: A web-2.0 based system for the preservation and presentation of cultural heritage. Computers \& Education, 53, pp. 841-852.

Cabrera, S. y Camarero Calandria, M.E. (2016). Comunicación de la ciencia y la tecnología en las universidades ecuatorianas. Estudio preliminar del impacto y percepción entre la población universitaria. Revista de la SEECI, 40, pp. 27-47. Recuperado de https://goo.gl/JDaPUv

Caro Valverde, M.T. (2018). La comunicación argumentativa en la Sociedad del Conocimiento, clave del liderazgo distribuido para un cambio educativo desde el desarrollo profesional. RED. Revista de Educación a Distancia, 56. Recuperado de https://goo.gl/L76ZJE

Carretero, S.; Vuorikari, R. and Punie, Y. (2017). DigComp 2.1: The Digital Competence Framework for Citizens with eight proficiency levels and examples of use. Recuperado de https://goo.gl/F2EwJC

Colás-Bravo, P., Conde-Jiménez, J. y Martín-Gutiérrez, Á. (2015). Las redes sociales en la enseñanza universitaria: Aprovechamiento didáctico del capital social e intelectual. Revista Interuniversitaria de Formación del Profesorado, 29, (2).

Chaín Navarro, C., Martínez Solís, L. y Sánchez Baena, J.J. (2008). Motivar desde la innovación en la enseñanza universitaria: El blog qalidad. RED. Revista de Educación a Distancia, 21. Recuperado de https://goo.gl/RMd3oq

Chaín Navarro, C. (2013). Repositorios digitales: un universo infinito de recursos para los docentes e investigadores del área de Humanidades. En E. Castro Páez, P. Cervera Corbacho y A.M. Bocanegra Valle (Coords.). Historia y desafíos de la edición en el mundo hispánico: II Foro editorial de estudios hispánicos y americanistas. Vol. I. Cádiz: Universidad de Cádiz, Servicio de Publicaciones, pp. 115-152.

Churchill, D. (2009). Educational applications of Web 2.0: Using blogs to support teaching and learning. British Journal of Educational Technology, 40 (1), pp. 179-183.

Dapía Conde, M. D. y Escudero Cid, R. (2014). Aprendizaje colaborativo mediante uso de blog en la enseñanza universitaria. Valoración de una experiencia. Enseñanza \& Teaching, 32(2), pp. 53-72. Recuperado de https://goo.gl/6zXRls

Esteve, F., Castañeda, L. y Adell, J. (2018). Un modelo holístico de competencia docente para el mundo digital. Revista Interuniversitaria de Formación del Profesorado, 32(1).

Fernández Fernández, L. (2015). MUPATEC. Plataforma virtual para el proceso de enseñanza/aprendizaje de las asignaturas de Museología, Patrimonio Cultural, y

Humanidades digitales para el aprendizaje y difusión del Patrimonio Naval. Lorena Martínez-Solís y Celia Chaín-Navarro

Página 15 de 19 
Técnicas Artísticas (III) (Proyecto de Innovación y Mejora de la Calidad Docente). Universidad Complutense de Madrid.

Goktas, Y. (2009). Incorporating Blogs and the Seven Principles of Good Practice into Preservice ICT Courses: A Case Study. The New Educational Review, 19(3-4), pp. 2944. Recuperado de https://goo.gl/bj8VY3

Gómez, M.; Roses, S. y Farias, P. (2012). El uso académico de las redes sociales en universitarios. Comunicar, XIX(38), pp. 131-138.

González Bencomo, H. y Delgado Acosta, C.R. (2016). Facebook: herramienta didáctica para el aprendizaje de la geografía. Revista DIM: Didáctica, Innovación y Multimedia, 34. Recuperado de https://goo.gl/8XuKjp

González Martínez, J., Lleixa Fortuño, M. y Espuny Vidal, C. (2016). Las redes sociales y la educación superior. Las actitudes de los estudiantes universitarios hacia el uso educativo de las redes sociales, de nuevo a examen. Education in the knowledge society (EKS), 17(2), pp. 21-38. Recuperado de https://goo.gl/vpqMia

González Ramírez, M.R. (2016). DUENDE. Docencia Universitaria en Dirección de Empresas. En J.D. Álvarez Teruel, S. Grau Company y M.T. Tortosa Ybáñez (Coords.). Innovaciones metodológicas en docencia universitaria: resultados de investigación. Alicante: Universidad de Alicante, pp. 733-746. Recuperado de https://goo.gl/7gVVgU

Gros, B. (2012). Retos y tendencias sobre el futuro de la investigación acerca del aprendizaje con tecnologías digitales. RED, Revista de Educación a Distancia, 32. Recuperado de https://goo.gl/xhw4sS

Gross, A.G. y Harmon, J.E. (2016). The internet revolution in the sciences and humanities. Oxford; New York: Osford University Press.

Halic, O. [et al.] (2010). To blog or not to blog: Student perceptions of blog effectiveness for learning in a college-level course. Internet and Higher education, 13, pp. 206-213. Recuperado de https://goo.gl/HTkyQt

Hernández-Ramos, P. (2004). Web logs and online discussions as Tools to Promote Reflective Practice. The Journal of Interactive Practice, 3(1), pp. 1-16. Recuperado de https://goo.gl/ZBHg6Q

Iribarren i Donadeu, T (2017). Las Humanidades Digitales. En Fuentes especializadas en Ciencias Sociales y Humanidades. Madrid: Ediciones Pirámide, pp. 441-473.

Jiménez Migallón, A. [et al.]. (2016). Nuevas estrategias de aprendizaje para trabajos fin de grado en Ciencia de los Alimentos. En M.T. Tortosa Ybáñez, S. Grau Company y J.D. Álvarez Teruel (Coords.), XIV Jornadas de Redes de Investigación en Docencia Universitaria. Investigación, innovación y enseñanza universitaria: enfoques pluridisciplinares, Alicante: Universidad de Alicante, pp. 1422-1439. Recuperado de https://goo.gl/69KYaZ

\footnotetext{
Humanidades digitales para el aprendizaje y difusión del Patrimonio Naval. Lorena Martínez-Solís y Celia Chaín-Navarro

Página 16 de 19
} 
Krause, S.D. (2005). Blogs as a Tool for Teaching. The Chronicle of Higher Education. Information Technology, 51(42), B33-B35. Recuperado de https://goo.gl/0FlzKn

Linares, N. [et al.]. (2016). Análisis de herramientas TICs de contenido científico para la docencia en Ciencias Experimentales. En M.T. Tortosa Ybáñez, S. Grau Company y J.D. Álvarez Teruel (Coords.), XIV Jornadas de Redes de Investigación en Docencia Universitaria. Investigación, innovación y enseñanza universitaria: enfoques pluridisciplinares, Alicante: Universidad de Alicante, pp. 585-601. Recuperado de https://goo.gl/69KYaZ

Maquilón Sánchez, J.J., Mirete Ruiz, A.B. \& Avilés Olmos, M. (2017). La Realidad Aumentada (RA). Recursos y propuestas para la innovación educativa. Revista Electrónica Interuniversitaria de Formación del Profesorado, 20(2), pp. 183-203.

Marcelo, C. [et al.]. (2016). Actividades de aprendizaje con tecnologías en la universidad. ¿Qué uso hacen los profesores? Profesorado: Revista de curriculum y formación del profesorado, 20(3), pp. 283-312. Recuperado de https://goo.gl/oOEvyH

Martínez Clares, P., Pérez Cusó y Martínez Juárez, M. (2016). Las TICs y el entorno virtual para la tutoría universitaria. Educación XX1: Revista de la Facultad de Educación, 19(1), pp. 287-310. Recuperado de https://goo.g1/B5VNy1

Martín Montilla, A. y Montilla Coronado, M.V.C. (2016). El uso del blog como herramienta de innovación y mejora de la docencia universitaria. Profesorado, revista de currículum y formación del profesorado, 20(3), pp. 659-686. Recuperado de https://goo.gl/f2q3il

Martínez Serrano, M.C. y Ferraz da Cunha, E. (2016). Uso de las redes sociales por los alumnos universitarios de educación. Un estudio de caso de la península ibérica. Tendencias pedagógicas, 28, pp. 33-44. Recuperado de https://goo.gl/ms3Rp4

Martínez Solís, L., Chaín Navarro, C. y Sánchez Baena, J.J. (2011). Continuar aprendiendo sin coincidir ni en el espacio ni el tiempo: propuesta para la asignatura "Archivística, Documentación y Tecnología" del Posgrado en Historia y Patrimonio Naval. En Congreso Internacional de Innovación Docente, Cartagena: Universidad Politécnica de Cartagena, pp.141-160. Recuperado de https://goo.gl/nxTCLU

Martínez Solís, L., Chaín Navarro, C. y Sánchez Baena, J.J. (2014). Aprendiendo a difundir el Patrimonio Marítimo en la web 2.0 a través del Máster de Historia y Patrimonio Naval. Revista Internacional de Tecnologías en la Educación, 1(2), pp. pp. 25-40. Recuperado de https://goo.gl/2uA3E3

Molina Alventosa, P. [et al.] (2013). Uso de blogs y evaluación continua del aprendizaje del alumnado universitario. EDUTEC, Revista Electrónica de Tecnología Educativa, 43. Recuperado de https://goo.gl/iCJEFl

Molina Alventosa, P., Valencia-Peris, A. y Gómez Gonzalvo, F. (2016a). Innovación docente en educación superior. Edublogs, evaluación formativa y aprendizaje colaborativo. Profesorado: revista de currículum y formación del profesorado, 20(2),

Humanidades digitales para el aprendizaje y difusión del Patrimonio Naval. Lorena Martínez-Solís y Celia Chaín-Navarro

Página 17 de 19 
pp. 432-450. Recuperado de https://goo.gl/bCi1PK

Molina Alventosa, P., Valencia-Peris, A. y Suárez Guerrero, C. (2016b). Percepción de los estudiantes de una experiencia de uso didáctico de blog docente en educación superior. Educación XX1: Revista de la Facultad de Educación, 19(1), pp. 91-113. Recuperado de https://goo.gl/7XTMZz

Morán López, P.M. (2016). E-learning 2.0 en el aula de ELE. Optimización metodológica de entornos telemáticos cooperativos y móviles como recursos didácticos del español como segunda lengua y lengua extranjera (tesis doctoral). Universidad de Oviedo.

Onrubia, J. (2016). Aprender y enseñar en entornos virtuales: actividad conjunta, ayuda pedagógica y construcción del conocimiento. RED-Revista de Educación a Distancia, 50. Recuperado de https://goo.gl/bQEqjn

Payá Rico, A. y Álvarez Domínguez, P. (2016a). Història de 1’Educació 2.0 i Història de l'e-Educació. Les TIC i les xarxes socials al servei de la docència historicoeducativa. Educació i historia: Revista d'història de l'educació, 26, pp. 41-64. Recuperado de https://goo.gl/Xa8DKS

Payá Rico, A., Duart Montoliu, J.M. y Mengual-Andrés, S. (2016b). Histoedu, redes sociales e historia de la educación. El pasado pedagógico desde el presente educativo. Education in the knowledge society (EKS), 17(2), pp. 55-72. Recuperado de https://goo.gl/qWlrdX

Prendes Espinosa, M.P.; Gutiérrez Porlán, I. y Martínez Sánchez, F. (2018). Competencia digital: una necesidad del profesorado universitario en el siglo XXI. RED. Revista de Educación a Distancia, 56. Recuperado de https://goo.gl/gwj1nG

Reigeluth, C. (2012). Instructional Theory and Technology for the New Paradigm of Education. RED, Revista de Educación a Distancia, 32. Recuperado de https://goo.gl/O7mYxS

Rivero Panaqué, C. [et al.]. (2016). Las TIC en la formación universitaria. Logros y desafíos para la formación en psicología y educación. Revista de Psicología, 34(1), pp. 185-199. Recuperado de https://goo.gl/aY4lwK

Romero-Frías, E. y Sánchez González, M. (eds.) (2014). Ciencias sociales y humanidades digitales. Técnicas, herramientas y experiencias de e-Research e investigación en colaboración. Cuadernos Artesanos de Comunicación, 61. Recuperado de https://goo.gl/vzhQkk

Rojano Ramos, S., López Guerrero, M.M. y López Guerro, G. (2016). Desarrollo de tecnologías de la información y de la comunicación para reforzar los procesos de enseñanza y aprendizaje en ciencias del grado de maestro/a en educación infantil de la Universidad de Málaga. Educación Química, 27(3), pp. 226-232. Recuperado de https://goo.gl/iPITsB

Romero Frías, E. y Robinson García, N. (2017). Laboratorios sociales en universidades.

Humanidades digitales para el aprendizaje y difusión del Patrimonio Naval. Lorena Martínez-Solís y Celia Chaín-Navarro

Página 18 de 19 
Innovación e impacto en Medialab UGR. Comunicar: Revista científica iberoamericana de comunicación y educación, 51, pp. 29-38. Recuperado de https://goo.gl/WwYkBa

Rovira Collado, J. [et al.]. (2016). Redes sociales de lectura y otras herramientas de la LIJ 2.0 en asignaturas de didáctica de la lengua y la literatura de la Universidad de Alicante. En J.D. Álvarez Teruel, S. Grau Company y M.T. Tortosa Ybáñez (Coords.). Innovaciones metodológicas en docencia universitaria: resultados de investigación. Alicante: Universidad de Alicante, pp. 927-941. Recuperado de https://goo.gl/BOjgq4

Salgado Santamaría, C., González Conde, M.J. y Zamarra López, M.M. (2013). Innovación y aplicación tecnológica en el ámbito de la Educación Superior universitaria. El empleo de los blogs en las universidades españolas. Historia y Comunicación Social, $18, \mathrm{n}^{\mathrm{o}}$ especial diciembre, pp. 613-625. Recuperado de https://goo.gl/hCJpeK

Sánchez Rodríguez, C. [et al.]. (2016). Las redes sociales como herramientas de comunicación, debate y evaluación del trabajo en equipo. M.T. Tortosa Ybáñez, S. Grau Company y J.D. Álvarez Teruel (Coords.), XIV Jornadas de Redes de Investigación en Docencia Universitaria. Investigación, innovación y enseñanza universitaria: enfoques pluridisciplinares, Alicante: Universidad de Alicante, pp. 1610-1626. Recuperado de https://goo.gl/69KYaZ

Suárez-Llorca C, Carrasco-Embuena V, Fourcade-López A, Chinchilla-Mira JJ, \& AndreuCabrera E. (2010). The effects of the use of blogs on the learning process of sea sports in the framework of higher education. Journal of Human Sport and Exercise; 5(2), pp. 288-294.

Top, E. (2012). Blogging as a social medium in undergraduate courses: Sense of community best predictor of perceived learning. Internet and Higher Education, 15(1), pp. 24-28. Recuperado de https://goo.gl/1Y5cv9

Valverde-Crespo D., Pro-Bueno A. y Gónzález-Sánchez, J. (2018) La competencia informacional-digital en la enseñanza y aprendizaje de las ciencias en la educación secundaria obligatoria actual: una revisión teórica. Revista Eureka sobre Enseñanza y Divulgación de las Ciencias 15(2). Recuperado de https://goo.gl/be4JjK

Wong, L.H., Shing, C. y AW, G.P. (2017). Aprendizaje de idiomas "sin costuras". Aprendizaje de segundas lenguas y redes sociales. Comunicar: Revista científica iberoamericana de comunicación y educación, 50, pp. 9-21. Recuperado de https://goo.gl/hzMUWc

Humanidades digitales para el aprendizaje y difusión del Patrimonio Naval. Lorena Martínez-Solís y Celia Chaín-Navarro

Página 19 de 19 